\title{
(6) OPEN ACCESS \\ Time for change: a roadmap to guide the implementation of the World Anti-Doping Code 2015
}

\author{
Jiri Dvorak, ${ }^{1}$ Norbert Baume, ${ }^{2}$ Francesco Botré, ${ }^{3}$ Julian Broséus, ${ }^{4}$ Richard Budgett, ${ }^{5}$ \\ Walter 0 Frey, ${ }_{1}^{6}$ Hans Geyer, ${ }^{7}$ Peter Rex Harcourt, ${ }^{8}$ Dave Ho, ${ }^{9}$ David Howman, ${ }^{10}$ \\ Victor Isola ${ }_{1}^{11}$ Carsten Lundby, ${ }_{12}^{12}$ François Marclay, ${ }_{1}^{13}$ Annie Peytavin, ${ }_{1}^{14}$ \\ Andrew Pipe, ${ }^{15}$ Yannis P Pitsiladis, ${ }^{16}$ Christian Reichel, ${ }^{17}$ Neil Robinson, ${ }^{18}$ \\ Grigory Rodchenkov, ${ }^{19}$ Martial Saugy, ${ }^{20}$ Souheil Sayegh, ${ }^{21}$ Jordi Segura, ${ }^{22}$ \\ Mario Thevis, ${ }^{23}$ Alan Vernec, ${ }^{24}$ Marjolaine Viret, ${ }^{25,26}$ Marc Vouillamoz, ${ }^{27}$ \\ Mario Zorzolii
}

For numbered affiliations see end of article.

\section{Correspondence to} Professor Jiri Dvorak, FIFA/F-MARC FIFA-Strasse Zurich 8048, Switzerland; jiri.dvorak@f-marc.com

Received 11 February 2014 Accepted 14 February 2014

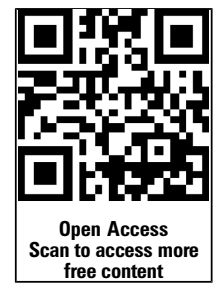

CrossMark

To cite: Dvorak J,

Baume N, Botré $\mathrm{F}$, et al. Br J Sports Med 2014:48. 801-806.

\section{ABSTRACT}

A medical and scientific multidisciplinary consensus meeting was held from 29 to 30 November 2013 on Anti-Doping in Sport at the Home of FIFA in Zurich, Switzerland, to create a roadmap for the implementation of the 2015 World Anti-Doping Code. The consensus statement and accompanying papers set out the priorities for the antidoping community in research, science and medicine. The participants achieved consensus on a strategy for the implementation of the 2015 World Anti-Doping Code. Key components of this strategy include: (1) sport-specific risk assessment, (2) prevalence measurement, (3) sport-specific test distribution plans, (4) storage and reanalysis,

(5) analytical challenges, (6) forensic intelligence,

(7) psychological approach to optimise the most deterrent effect, (8) the Athlete Biological Passport (ABP) and confounding factors, (9) data management system (Anti-Doping Administration \& Management System (ADAMS), (10) education, (11) research needs and necessary advances, (12) inadvertent doping and (13) management and ethics: biological data. True implementation of the 2015 World Anti-Doping Code will depend largely on the ability to align thinking around these core concepts and strategies. FIFA, jointly with all other engaged International Federations of sports (Ifs), the International Olympic Committee (IOC) and World Anti-Doping Agency (WADA), are ideally placed to lead transformational change with the unwavering support of the wider antidoping community. The outcome of the consensus meeting was the creation of the ad hoc Working Group charged with the responsibility of moving this agenda forward.

\section{INTRODUCTION}

A medical and scientific multidisciplinary consensus meeting was held from 29 to 30 November 2013 on Anti-Doping in Sport at the Home of FIFA in Zurich, Switzerland, to create a roadmap for the implementation of the 2015 World Anti-Doping Code. The papers delivered at the consensus meeting are published in this themed edition of the BJSM along with the consensus statement. The consensus statement and accompanying papers set out the priorities for the antidoping community in research, science and medicine. This consensus is timed to coincide with the publication of the 2015 World Anti-Doping Code and brings together the latest scientific and medical evidence and reaffirms the commitment of science and medicine in the fight against doping in sport.

The fight against doping in sport was formally started by the International Olympic Committee (IOC) in the 1960s, culminating in the creation of World Anti-Doping Agency (WADA) in 1999. The antidoping movement is now poised to take a major step forward in the fight against doping in sport by implementing the new 2015 World Anti-Doping Code and drawing on the major advances in science and medicine, much of which are underpinned by research funded by WADA and the International Federations of sports (IFs). As such, evidence-based, targeted, sport-specific and situation-specific strategies along with more effective analysis and improved efficiency and intelligence are approaches envisaged to lead to better deterrence of doping in sport. These strategies, combined with education and the commitment of all antidoping organisations (ADOs) to implement evidence-based programmes, should help protect the integrity of sport and all those athletes who do not dope.

\section{Sport-specific risk assessment}

The overall strategy in the fight against doping must be based on good scientific evidence, statistical analysis reflecting the prevalence of doping cases and the monitoring of illicit substances. This assessment must be sport specific as the risk and temptation to dope and the doping strategy will depend largely on the type of sport. ${ }^{1}$ For example, within individual sports, endurance athletes in track and field, cycling or cross-country skiing would choose different substances and methods to illicitly improve their performance, in contrast to athletes who depend primarily on strength and power, such as weight-lifters, wrestlers or athletes in certain track and field disciplines. The situation in team sports is likely to be different as results depend primarily on the collective team performance, albeit that individual athletes could still be 
tempted to dope to improve performance prospects for team selection. $^{2}$

The risk assessment must also take into account the timing during the season when peak performance is desirable and expected. The longer the preparation period for a particular event-the so-called 'out of competition' period - that may precede cycling tours or marathon running, the greater the likelihood of eluding doping controls and the temptation to illicitly augment performance during this training period. It is prudent, therefore, that the IFs along with the organisations of athletes consider sport-specific risk assessments. Such assessments should be reappraised regularly reflecting scientific evidence and information derived from forensic intelligence. The IFs, national antidoping organisations (NADOs), doctors, scientists and paramedic personnel, together with representatives from WADA and the WADA-accredited laboratories, must design and formulate the risk assessment by sharing information with the IFs, the IOC and other major event organisations. The role of WADA in this respect is to coordinate information exchange and alert the IFs and all involved stakeholders of new information on possible doping substances, methods or approaches that could potentially help uncover doping.

\section{Prevalence measurement}

A recommended approach for defining the scope of the antidoping activity is to measure the prevalence of doping. The use of appropriate epidemiological tools and the careful interpretation of survey results with an understanding of the population examined will allow the extent of the doping problem to be assessed. ${ }^{3}$ This can be achieved by conducting questionnaire surveys as is commonly used in social sciences. Epidemiological studies can be further enhanced by measuring putative biomarkers of doping in doping-control samples. It is essential to carry out doping prevalence studies in a population of competitors, and to appropriately interpret the data before defining and implementing a programme of longitudinal follow-up. This is particularly important because of the specificity of different sports, which can be influenced differently by doping depending on the physical and physiological characteristics required for performance enhancement.

In public health, pandemics are not distributed evenly around the globe, and environmental, social and economic factors play an important role in their distribution. The same is true in sport where doping prevalence may vary as a function of the sport and the country in which it is practiced. Research recently conducted in track and field demonstrated that the prevalence of abnormal blood profiles can vary from 3\% to $48 \%$ depending on the country of origin of the athletes. ${ }^{3}{ }^{4}$ Certain sports federations, sports disciplines and even countries may fear total transparency in examining their 'doping cultures' as acknowledgement may hurt their public image. However, a transparent approach is necessary if the biological monitoring of athletes is to become an effective tool in the fight against doping.

\section{Sport-specific test distribution plans}

The 2015 World Anti-Doping Code makes specific reference to the development of test distribution plans and the necessity for thoughtful and strategic approaches to testing. ${ }^{5}$ Such approaches will ensure that the effective, intelligent and most efficient testing strategies are adopted. Fundamental to these considerations are the identification of areas of sport that might be deemed to be high risk for doping practices. International Federations and NADOs will have particular responsibilities in this respect.
Several elements should feature prominently in the development and preparation of a sport-specific testing programme. These include consideration of the unique subcultures of sport and the degree to which doping may have been 'normalised' within such subcultures, the history of doping practices within a sport, the specific physiological demands of particular events, emerging training practices, the competitive schedule, recovery from injury, an awareness of dramatic changes in performance, an understanding of the supplement marketplace and a familiarity with what is happening 'on the street' as well as 'in the stadium'.

Continual, ongoing conversation with athletes, coaches and others in the sport community can provide an enriched understanding of the likelihood of doping behaviour and emerging problematic practices. Successful antidoping programmes of the future will embody high-quality, intelligent testing practices rather than high-quantity test volumes.

The implementation of the new World Anti-Doping Code with the emphasis on more intelligent testing affords a great opportunity for enhanced, more effective and more efficient approaches to doping control. The Athlete Biological Passport (ABP) represents a further opportunity to ensure strategic and more focused testing. Implicit in the new approaches to doping control is the necessity for strategic relationships between and among ADOs at every level. Agreements between IFs, NADOs and major event organisers addressing shared approaches to results management and testing strategies-particularly as they apply to competitors who are part of ABP programmes-will benefit the antidoping movement.

The perspectives, experiences and strategies of IFs and NADOs can be integrated so as to permit more timely and costefficient testing, the sharing of intelligence regarding doping practices and doping practitioners and heightened vigilance of customs and other civil authorities with regard to the importation and distribution of prohibited substances particularly at the time of major sporting events. These approaches will benefit from the developing international 'community of practice' represented by leading IFs and NADOs; the development of that community will itself be stimulated by the growth of strategic partnerships and cooperative antidoping activities.

\section{Storage and reanalysis}

New peptides or designer drugs may be used by athletes who feel that there are currently no reliable analytical tests available. However, the 2015 World Anti-Doping Code allows for the storage of samples for up to 10 years, which markedly transforms the antidoping environment. The deterrent effect of delayed testing with newly devised analytical methods is substantial. It is important that ADOs implement this process; the new 2015 International Standard on Testing and Investigations sets out the requirements for $\mathrm{ADOs}$ to test, store and reanalyse samples. The IFs and other ADOs must prioritise which samples should be stored from which competitions on the basis of their risk assessments. Such decisions should, to the extent permissible under applicable laws, remain confidential to optimise the deterrent and detection elements of this new approach to doping control. It is important that the storage of samples be conducted in a manner that enables future analysis with methods that may not yet be fully developed or operational. An example would be the future analysis of molecular signatures of doping (see article by Pitsiladis et al). ${ }^{6}$ It is also imperative that samples are stored in a manner that protects the integrity of biological samples and the antidoping process, having due regard 
to legal requirements surrounding handling of human biological materials and related data. ${ }^{5}$

\section{Analytical challenges}

The continuously growing knowledge in medicine, molecular biology, biochemistry and biotechnology has substantially expanded the options to pharmacologically manipulate the performances of athletes. Unsurprisingly, this has resulted in the suspected and proven misuse of a wide range of peptide hormones and substances such as insulin, insulin-like growth factor 1 , human growth hormone (hGH), epoetins, chorionic gonadotrophins, gene doping substances including RNA interference (RNAi), 'designer drugs' (eg, Tetrahydrogestrinone) as well as non-approved, emerging or discontinued compounds (eg, aminoimidazolecarboxamideriboside-AICAR, GW1516, selective androgen receptor modulators, hypoxia-inducible factor stabilisers and erythropoietin (Epo)-mimetic agents). These developments represent a considerable analytical challenge for antidoping scientists, which require dedicated research and the development of new methodologies. Concerted activities with civil authorities are also necessary to understand and combat the changing scope of doping practices and products.

Analytically, the issue of new, discontinued, or 'tailored' drug entities has been successfully tackled by applying non-target/ open approaches, biomarker-based assays (eg, haematological, steroidal and endocrinological modules of the ABP), 'omics' strategies and monitoring effects of drug (mis)use rather than the administered drug. This has been carried out via proactive and retrospective monitoring programmes. Some recent successes include the introduction of section 'S.0' to the WADA Prohibited List, new detection methods for the determination of RNAi-based compounds in blood and urine, the analysis of Epo microdosing, adverse analytical findings for the non-approved drugs GW1516, andarine and ostarine, as well as new methods to detect the illicit use of natural compounds such as AICAR. ${ }^{7-9}$

\section{Forensic intelligence}

The importance of investigations is enhanced in the 2015 World Anti-Doping Code $^{10}$ to further encourage ADOs to pursue antidoping violations based on the strongest evidence possible. While investigations as the primary means of proving doping violations are uncommon in sport, investigations have been successful in highlighting doping practices and developing novel approaches in the fight against doping in sport.

High-profile investigations include the Bay Area Laboratory Co-Operative (BALCO) investigation that was undertaken in the USA in 2003.This case resulted in the prosecution of several athletes for the use of hGH and new designer steroids and led to new laboratory detection capabilities and changes to the World Anti-Doping Code. ${ }^{11} 12$ In 2006, a Spanish investigation code-named Operation Puerto highlighted serious doping practices involving Dr Eufemiano Fuentes and a number of elite athletes. ${ }^{13} 14$

In 2013, the Australian Crime Commission uncovered links between organised crime and professional sporting teams and the use of performance-enhancing 'peptides' and other illicit substances. ${ }^{15}$ This investigation led to a team in the Australian Football League being charged and subsequently sanctioned. ${ }^{16}$ At the heart of this case were the activities of support staff. Therefore, it follows that close scrutiny of support staff is explicit in the 2015 World Anti-Doping Code. The successful conclusion of each of these highlighted cases relied on forensic science and other modern investigative techniques now at the disposal of IFs and NADOs and in accordance with the investigation aspects of the 2015 World Anti-Doping Code.

A forensic intelligence model of antidoping investigations was proposed by Marclay et $a l^{17}$ and included broader exploitation of information held at different levels by antidoping partners such as the police, borders agencies and postal services, strategic Internet monitoring, physical and chemical drug profiling and doping script analysis within a forensic intelligence framework. Tactical use of forensic intelligence tools relies largely on the exploitation of bioanalytical results, documents linked to doping practice and seizures of prohibited substances. At the operational level, the exploitation of such information serves to uncover trends related to the abuse of prohibited substances, the existence of organised doping programmes including the trafficking of doping agents, and helps identify their structure and mechanisms of operation. Strategic Internet monitoring also allows the identification and monitoring of online sales websites, forums, blogs, social networks and other online media, thus helping to create a clearer picture of the market and emergence of new trends. ${ }^{18}$ Monitoring of the physical and chemical profiles of seized products further enhances the understanding of the organisational structure of the trafficking of prohibited substances. ${ }^{19}$ Drug profiling as well as digital and other data allow the building of 'inference models' that can link to product seizures, highlight distribution networks and identify the sources of supply. Finally, script analysis can map the complete sequence of activities before, during and after doping to identify the key stages and possible intervention points where the doping process might be disrupted or even prevented. ${ }^{20}$

\section{Psychological approach to optimise most deterrent effect}

The perception of the likelihood of detection, the severity of the penalty and the speed with which sanctions will be applied all appear to deter doping behaviour. ${ }^{21}$ Better understanding of doping deterrents will enhance doping control programmes and reinforce the need for testing strategies to be carefully considered, strategically applied and robustly enforced.

The sense of 'right and wrong' and the perception of normative behaviour within a sport community are perhaps the most fundamental determinants of appropriate sporting behaviour. ${ }^{22-24}$ Doping practices have been mostly embedded in sports in which it was widely understood that such behaviours were part of the sport 'culture'. Therefore, sport organisations should consistently emphasise that drug-taking behaviour is fundamentally contrary to the principles and precepts of sport, that is, against the spirit of sport.

Sport can profoundly mould and modify attitudes and beliefs. The clear, unequivocal expression of a set of expectations regarding conduct and behaviour within a sport can have a powerful and enduring impact. The degree to which these expectations are upheld by athletes can enhance their legitimacy in the eyes of their fellow competitors and strengthen the perception that violations of such expectations are wrong. The profound disapproval that follows the violation of broadly valued standards of behaviour can be an immensely powerful sanction and the desire to avoid such disapproval an equally potent deterrent. Therefore, the creation of what has been described as a 'moral cosmology' and an associated 'moral community' is central to the development of a sporting community in which doping practices are reduced to an absolute minimal level (accepting that there will always be those who succumb in sport, as elsewhere, to the temptation to cheat). ${ }^{21} 25$ 


\section{The ABP and confounding factors}

Typical doping control based on the direct detection of a substance or its metabolites is an effective approach. However, it has limitations particularly when an athlete may be using substances on an intermittent and/or low-dose basis, which may therefore go undetected under even the most robust In-of-Competition and Out-of-Competition Doping Control programme. Furthermore, the availability of substances virtually identical to those produced by the human body, such as the native form of Epo, testosterone and growth hormone necessitated a new drug-testing paradigm.

Longitudinal profiling, which eventually became harmonised into the scientifically robust WADA ABP programme, is a complementary and alternative means to traditional doping control. Doping leaves a characteristic 'fingerprint' on the biology of the athlete and the ABP is used to identify that fingerprint, and thus the occurrence of doping. Once a biomarker of doping is implemented in the ABP, the potential to detect those changes brought on by performance-enhancing drugs is increased; it may prove possible to detect changes caused by substances that have not yet been identified. ${ }^{26}$

The intelligent and timely interpretation of ABP data can lead to target testing for specific substances. Alternatively, an atypical passport finding which is confirmed by an Expert Panel can lead to an athlete being charged with an antidoping rule violation without a 'positive' test (Adverse Analytical Finding). Thus, the $\mathrm{ABP}$ can be seen as an innovative and reliable antidoping tool as reflected by the findings of Court of Arbitration for Sport panels in several cases. The introduction of the ABP also provides a strong doping deterrent and a boost to the credibility of the fight against doping in any given sport. ${ }^{27}$ The ABP does not only involve the monitoring of biological markers. Confounding factors such as age, sex and exposure to higher altitude for the haematological module are also included in the passport for improved decision-making. ${ }^{28}$ Several confounding factors are also described in the WADA 2014 endogenous anabolic steroids technical document (TDEAAS2014). ${ }^{29}$ Detailed information regarding sample collection, transport and analysis is included in the technical documents that accompany the WADA ABP Operating Guidelines. ${ }^{28}$

\section{Data management system-Anti-Doping Administration \& Management System}

Regardless of advancements in science and enhanced antidoping practice and policy, the fight against doping in sport can only succeed if there is a coordinated effort to ensure that the limited resources are used effectively. In this regard, the collection, analysis and sharing of doping control-related information and intelligence are imperative. Only by using a single database to collect and disseminate such information can the global antidoping community intelligently coordinate their efforts. Anti-Doping Administration \& Management System (ADAMS) provided by WADA, adapts to support the ever-changing antidoping environment. A single database also ensures consistency in protecting the rights of athletes vis-à-vis their information and ever-emerging data protection best practices.

\section{Education}

One of the objectives of successful antidoping education is to ensure that all those involved in sport understand the harm caused by doping to the health of athletes and to the integrity and essence of sport. As all sport-related stakeholders have a role to play to promote clean sport, educational efforts must be inclusive of the broad sporting community including athletes, coaches, physicians, teachers and parents. This objective will require the commitment of the IOC, IFs, governments (to reach schools and community-level sport) and NADOs, with WADA as the coordinator.

Education should be ongoing and sustained; it must take place throughout the entire sporting career of an individual and focus on values and good decision-making skills as well as an appreciation of the roles and responsibilities of athletes. The education of athletes has to start as early as possible, preferably prior to an athlete's first national/international competition. For example, FIFA introduced a standard educational programme to all participating teams of the FIFA U-17 World Cup (boys and girls) 2012 that was overseen by doctors responsible for the competition. This is in addition to other grassroot education programmes already implemented by organisations such as UEFA that involve the education of more than 1000 young international football players each year. For the success of these programmes, the message presented to athletes needs to be clear and at the correct level. The use of posters, to decorate the typically 'unfriendly' surroundings, and advice cards could also facilitate such communication.

\section{Research needs and necessary advances}

Approaches to detect doping have improved significantly in recent years but remain imperfect and therefore new direct and indirect detection methods are required. New integrative 'Omics'-based solutions are being developed that have the potential to improve the analytical performance of current detection methods. ${ }^{30}$ In particular, WADA is funding studies to identify a 'molecular signature' of recombinant human erythropoietin (RhumanEpo) doping and preliminary results are promising. ${ }^{31}$ For example, in the first systematic study to be conducted, the expression of hundreds of genes were found to be altered by RhumanEpo with numerous gene transcripts being differentially expressed after the first injection and further transcripts profoundly upregulated during and subsequently downregulated up to 4 weeks postadministration of the drug, with the same transcriptomic pattern observed in all participants. The identification of a blood 'molecular signature' of RhumanEpo administration is the strongest evidence to date that gene biomarkers have the potential to substantially improve the analytical performance of current antidoping methods such as the ABP for RhumanEpo detection. These encouraging results serve to strongly reinforce the feasibility and need for this complex, expensive and technically demanding approach involving leading industry partners for the detection of banned substances and methods. Therefore, research using an 'omics'based approach involving genomics, transcriptomics, proteomics and metabolomics should be greatly intensified in order to achieve improved detection of Recombinant human Epo and other doping substances and methods difficult to detect such as growth hormone and blood transfusions.

\section{Inadvertent doping}

A major objective of the global fight against doping is the protection of the clean athlete, and hence the need to inform athletes of the risks of inadvertent doping. In recent years, antidoping research has identified contaminated nutritional supplements and food as the principal sources of inadvertent doping. Nutritional supplements have been contaminated with various stimulants, $\beta 2$-agonists, prohormones, 'classic' anabolic steroids and non-approved designer steroids. ${ }^{32} 33$ 
Similarly, the consumption of certain foods, especially meat products, is of particular concern as they may result in an adverse analytical finding. Recent investigations have shown that the anabolic agent clenbuterol is misused in some countries as a growth promoter in cattle feeding. The consumption of meat from clenbuterol-treated cattle may lead to adverse analytical findings and to poisoning cases. ${ }^{34}$ WADA has communicated with specific governments to address this concern as it relates to doping and sport. WADA and FIFA are also working on studies that may differentiate the source of clenbuterol to detect whether the substance found in the body may be due to an indirect ingestion from an animal product. First promising results have already been obtained. ${ }^{36} 37$ The identification of such 'doping traps' and the dissemination of the knowledge of such sources of inadvertent doping to all stakeholders are important aspects in the protection of athletes.

\section{Management and ethics: biological data}

The introduction of the ABP is a reason to consider expanding the role of the medical profession in the fight against doping in sport. The ABP is a recently validated approach to the identification and prosecution of doping rule violations. The routine implementation of the ABP may result in the inadvertent identification of potential clinical situations and this will need to be addressed by medical experts. When alerted to laboratory findings, physicians are obliged to inform the athlete via the ADOs if there is a suspected pathology. ADOs, particularly the IFs and the NADOs, must respond accordingly to ensure the appropriate involvement of medical professionals in the process of individual case management. At present, the mandate of ADOs is primarily to ensure that the health of the athlete is not affected by entering a doping spiral rather than as a health check system, and the process typically does not involve a physician. New rules would be required to address this issue. ${ }^{5}$

Whenever doctors affiliated with ADOs are involved in results management, they must ensure that anomalous results of potential clinical significance are investigated appropriately. In such situations, communication with other physicians involved in the care of the particular athlete outlining the findings, their implication and suggesting, when necessary, an approach to their further investigation has been a common approach. It is unreasonable to expect that non-physicians would have the training, experience or perspective to assume responsibility for such a process. The management of such cases requires distinct medical knowledge and clinical experience.

The introduction of the ABP has raised a number of questions that reflect a profound concern for issues of confidentiality and the responsibility of those in receipt of biological information to take action if and when information that may relate to the health of a competitor becomes apparent. There may be significant national considerations to take into account in such circumstances. In some jurisdictions, testing authorities and laboratories are not considered or accredited as providing healthcare-related services and the disclosure of information emanating from such facilities, subsequently used for clinical purposes, may jeopardise such accreditation. Clearly, there is no expectation that analytical laboratories should see their role as expanding into 'clinicopathological' domains. The identification of anomalous findings by a clinician should be seen as an en passant phenomenon occasioned by a clinician's review of antidoping laboratory results and prompting further clinicalstandard investigations. The activities of clinicians in this respect should not be misinterpreted as evidence that antidoping analyses reflect clinical activities on the part of the antidoping laboratory.

However, clinicians would argue that they have a fundamental ethical responsibility to take action when provided with information that may reflect an underlying pathological condition. Physicians are obliged to alert the ADOs if they note anomalous results that are suspicious of pathology. The assessment of results by laboratory and medical experts (as part of the ABP) is carried out anonymously, therefore contact must be made via the ADO. Notably, in urine samples from male athletes, an elevation of the levels of human choriogonadotropin hormone (hCG) is quite common with approximately 90 cases a year according to WADA statistics. ${ }^{38}$ Elevated hCG may be due to the intake of the exogenous hormone but could reflect an underlying pathology-most typically testicular cancer. For all such cases, a specialised medical examination must be recommended as soon as possible to ensure appropriate investigation and treatment. Physicians experienced in providing oversight to antidoping programmes are familiar with this scenario and understand the importance of their intervention to ensure that proper clinical attention is given to the athletes concerned. WADA has provided clear instructions to ADOs to contact athletes to seek further medical investigation when elevated hCG levels are detected. There are a number of documented cases where such early intervention has led to a complete cure of the underlying condition and can be seen as an extremely positive aspect of doping control activities. hCG testing is part of routine analytical doping tests and not part of the ABP.

Equally challenging is the question of the right of competitors to have access to their test results. This is a complex and highly problematic issue given that such access may allow doping competitors to manipulate or modify their strategies so as to be more likely to escape detection. Furthermore, national legislation may, in many jurisdictions, mandate the release of such information; the question of the timing of such release may be critically important in ensuring the integrity of the testing system. These challenges notwithstanding, the importance to protect the data accumulated in the conduct of doping control programmes is paramount. It is important for sport organisations and their officials and staff to understand the robust and rigorous approaches that are used to safeguard personal health and related biological information in other community settings.

\section{Summary and conclusions}

The purpose of this paper was to summarise the results of the consensus meeting on Anti-Doping in Sport sponsored by FIFA. The participants achieved consensus on a strategy for the implementation of the 2015 World Anti-Doping Code. Key components of this strategy include: (1) sport-specific risk assessment, (2) prevalence measurement, (3) sport-specific test distribution plans, (4) storage and reanalysis, (5) analytical challenges, (6) forensic intelligence, (7) psychological approach to optimise most deterrent effect, (8) ABP and confounding factors, (9) data management system (ADAMS), (10) education, (11) research needs and necessary advances, (12) inadvertent doping and (13) management and ethics: biological data. True implementation of the 2015 World Anti-Doping Code will depend largely on the ability to align thinking around these core concepts and strategies. FIFA, jointly with all other engaged IFs, the IOC and WADA are ideally placed to lead transformational change with the unwavering support of the wider antidoping community. The outcome of the consensus meeting was the creation of the ad hoc Working Group charged with the responsibility of moving this agenda forward. 
Author affiliations

${ }^{1}$ FIFA/F-MARC FIFA-Strasse, Zurich, Switzerland

${ }^{2}$ Lausanne Anti-Doping Laboratory, Epalinges, Switzerland

${ }^{3}$ Department of Experimental Medicine, Sapienza University of Rome, Rome, Italy

${ }^{4}$ School of Criminal Sciences, University of Lausanne, Lausanne, Switzerland

${ }^{5}$ International Olympic Committee, Lausanne, Switzerland

${ }^{6}$ International Floorball Federation, Helsinki, Finland

${ }^{7}$ WADA Accredited Laboratory, German Sport University, Cologne, Germany

${ }^{8}$ Department of Integrity, AFL, Melbourne, Victoria, Australia

${ }^{9}$ International Rugby Board, Dublin, Ireland

${ }^{10}$ World Anti-Doping Agency (WADA), Montreal, Canada

${ }^{11}$ International Baseball Federation (IBAF), Lausanne, Switzerland

${ }^{12} \mathrm{ZIHP}$ and Institute of Physiology, University of Zurich, Zurich, Switzerland

${ }^{13}$ CHUV, Swiss Laboratory for Doping Analyses, Ch. des Croisettes22, Epalinges,

Switzerland

${ }^{14}$ International Volleyball Federation (FIVB), Lausanne, Switzerland

${ }^{15}$ Federation International de Natation (FINA), Lausanne, Switzerland

${ }^{16}$ Institute of Cardiovascular and Medical Sciences, Glasgow, University of Glasgow,

Glasgow, Lanarkshire, UK

${ }^{17}$ Anti-Doping Laboratory, SeibersdorfLabor, Seibersdorf, Austria

${ }^{18}$ CURML, LAD, Ch des Croisettes 22, Epalinges, Switzerland

${ }^{19}$ Antidoping Centre Moscow, Moscow, Russian Federation

${ }^{20}$ CURML, LAD, Epalinges, Switzerland

${ }^{21}$ International Basketball Federation (FIBA), Geneva, Switzerland

${ }^{22}$ Bioanalysis Research Group, IMIM, Carrer Dr.Aiguader 88, Barcelona, Barcelona,

Spain

${ }^{23}$ Institute of Biochemistry and Center for Preventive Doping Research, German Sport

University Cologne, Cologne, Germany

${ }^{24}$ WADA, Montreal, Quebec, Canada

${ }^{25}$ Law-Sports Law II, University of Neuchâtel, Neuchâtel, Switzerland

${ }^{26}$ Attorney-at-law-Rue Caroline46, Carouge, Geneva, Switzerland

${ }^{27}$ UEFA, Neuchatel, Switzerland

${ }^{28}$ Medical, UCl, Chemin de la Mêlée, Aigle, Switzerland

\section{Competing interests None.}

Provenance and peer review Not commissioned; externally peer reviewed.

Open Access This is an Open Access article distributed in accordance with the Creative Commons Attribution Non Commercial (CC BY-NC 3.0) license, which permits others to distribute, remix, adapt, build upon this work non-commercially, and license their derivative works on different terms, provided the original work is properly cited and the use is non-commercial. See: http://creativecommons.org/ licenses/by-nc/3.0/

\section{REFERENCES}

1 Saugy M, Robinson N, Grimm K, et al. Future of the fight against doping: risk assessment, biological profiling and intelligence testing. Forensic Sci Int 2011;213:1-2

2 Dvorak J. Current situations, challenges to implement Anti-Doping fight-need for change? Br J Sports Med 2014; this issue.

3 Saugy M, Robinson N, Saudan C. The fight against doping: back on track with blood. Drug Test Anal 2009;1:474-8.

4 Sottas PE, Robinson N, Fischetto $G$, et al. Prevalence of blood doping in samples collected from elite track and field athletes. Clin Chem 2011;57:762-9.

5 Viret M. How to make science and law work hand in hand in Anti Doping - a survey of some critical issues under the 2015 WADA Code. Br J Sports Med 2014; this issue.

6 Pitsilasis YP, Durussel J, Rabin O. An integrative 'Omics' solution to the detection of recombinant human erythropoietin and blood doping. Br J Sports Med 2014:48:856-61.

7 Thevis M, Kuuranne $\mathrm{T}$, Geyer $\mathrm{H}$, et al. Annual banned-substance review: analytical approaches in human sports drug testing. Drug Test Anal 2012;4:2-16.

8 Thevis M, Kuuranne T, Geyer $\mathrm{H}$, et al. Annual banned-substance review: analytical approaches in human sports drug testing. Drug Test Anal 2013;5:1-19.

9 Thevis M, Kuuranne T, Geyer $\mathrm{H}$, et al. Annual banned-substance review: analytical approaches in human sports drug testing. Drug Test Anal 2014;6:in press.

10 WADA 2015 Code: http://www.wada-ama.org/en/World-Anti-Doping-Program/ Sports-and-Anti-Doping-Organisations/The-Code/Code-Review/Code-Version-4-0/.
http://www.wada-ama.org/en/World-Anti-Doping-Program/Sports-and-Anti-DopingOrganisations/The-Code/Code-Review/Code-Version-4-0/ [cited 2013].

11 Kazlauskas R. Designer steroids. Handb Exp Pharmacol 2010;195:155-85.

12 Today U. Balco investigation timeline 2007. http://usatoday30.usatoday.com/sports/ balco-timeline.htm [cited 2013].

13 TAS 2009/A/1879-Alejandro Valverde Belmonte c. Comitaro Olimpico Nazionale Italiano, 2010.

14 TAS 2010/A/2070_Antidoping Schweiz v Jan Ullrich, 2012.

15 ACC Report 2013: http://www.crimecommission.gov.au/publications/other/ organised-crime-drugs-sport [cited 2013].

16 AFL Changes. 2013: http://www.afl.com.au/staticfile/AFL\%20Tenant/AFL/Files/ EssendonFC notice of charges.pdf [cited 2013].

17 Marclay $F$, Mangin $\bar{P}$, Margot $P$, et al. Perspectives for forensic intelligence in anti-doping: thinking outside of the box. Forensic Sci Int 2013:229:133-44.

18 Pazos D, Giannasi P, Rossy Q, et al. Combining Internet monitoring processes, packaging and isotopic analyses to determine the market structure: example of gamma butyrolactone. Forensic Sci Int 2013;230:29-36

19 Morelato M, Beavis A, Tahtouh M, et al. The use of forensic case data in intelligenceled policing: the example of drug profiling. Forensic Sci Int 2013;226:1-9.

20 Chiu YN, Leclerc B, Townsley M. Crime script analysis of drug manufacturing in Clandestine Laboratories: implications for prevention. Brit J Crimonol 2011;51:355-74

21 Strelan P, Boeckmann R. Why drug testing in elite sport does not work: perceptual deterrence theory and the role of personal moral beliefs. J App Soc Psych 2006:36:2902-34.

22 Foglia WD. Perceptual deterrence and the mediating effect of internalized norms among inner city teenagers. J Res Crime Delinquency 1997;34:414-43.

23 Nagin D, Pogarsky G. Integrating celerity, impulsivity and extralegal sanction threats into a model of general deterrence: theory and evidence. Criminology 2001:39:865-92

24 Paternoster R. The deterrent effect of the perceived certainty and severity of punishment: a review of the evidence and issues. Justice Q 1987:4:173-217.

25 Paternoster R, Simpson S. Sanction threats and appeals to morality: testing a rational choice model of corporate crime. Law Soc Rev 1996:30:549-83.

26 Sottas PE, Robinson N, Rabin 0, et al. The athlete biological passport. Clin Chem 2011:57:969-76.

27 Zorzoli M. The Athlete Biological Passport from the perspective of an anti-doping organization. Clin Chem Lab Med 2011;49:1423-5.

28 WADA Athlete Biological Passport operating guidelines \& compilation of required elements 2013. http://www.wadaama.org/Documents/Science Medicine/Athlete Biological_Passport/WADA-ABP-Operating-Guidelines_v4.0-EN.pdf [cited 2013].

29 WADA technical document for EAAS. 2014. http://www.wada-ama.org/Documents/ World_AntiDoping_Program/WADPLaboratories/Technical_Documents/WADATD2014-EAAS-Endogenous-Anabolic-Androgenic-Steroids-EN.pdf [cited 2013]

30 Reichel C. OMICS-strategies and methods in the fight against doping. Forensic SCi Int 2011;213:20-34

31 Pitsilasis YP, Durussel J, Rabin O. An integrative 'Omics' solution to the detection of recombinant human erythropoietin and blood doping. Br J Sports Med 2014 (this supplement).

32 Geyer H, Braun H, Burke LM, et al. Inadvertent Doping: A-Z of nutritional supplements: dietary supplements, sports nutrition foods and ergogenic aids for health and performance_-part 22. Br J Sports Med 2011:45:752-4.

33 Geyer H, Parr MK, Koehler K, et al. Nutritional supplements cross-contaminated and faked with doping substances. J Mass Spectrom 2008;43:892-902.

34 Guddat S, Fussholler G, Geyer H, et al. Clenbuterol-regional food contamination a possible source for inadvertent doping in sports. Drug Test Anal 2012;4: 534-8.

35 Thevis $M$, Geyer L, Geyer $\mathrm{H}$, et al. Adverse analytical findings with clenbuterol among U-17 soccer players attributed to food contamination issues. Drug Test Anal 2013:5:372-6.

36 Nicoli $R$, Petrou M, Badoud F, et al. Quantification of clenbuterol at trace level in human urine by ultra-high pressure liquid chromatography-tandem mass spectrometry. J Chromatogr A 2013;1292:142-50.

37 Thevis $M$, Thomas $A$, Beuck $S$, et al. Does the analysis of the enantiomeric composition of clenbuterol in human urine enable the differentiation of illicit clenbuterol administration from food contamination in sports drug testing? Rapid Commun Mass Spectrom 2013:27:507-12.

38 WADA Testing Figures 2013. http://www.wada-ama.org/Documents/Resources/ Testing-Figures [cited 2014]. 